\title{
5 year follow-up of a tricuspid valve replacement resulting from infective endocarditis complicated by multiple pulmonary abscesses
}

\begin{abstract}
A 30-year-old male intravenous drug user underwent a tricuspid valve replacement with a mechanical ATS Open Pivot ${ }^{\circledR}$ bileaflet prosthesis due to Staphylococcus aureus infective endocarditis. Preceding investigations also revealed concomitant bilateral pulmonary abscesses which were successfully treated with broad-spectrum antimicrobials. Follow-up at 5years demonstrated as well functioning valve prosthesis with a low gradient. This case emphasises the importance of a multidisciplinary approach in the successful treatment of infective endocarditis.
\end{abstract}

Keywords: infective endocarditis, intravenous drug user, lung abscess, staphylococcus aureus, long-term result
Volume 2 Issue I - 2015

\author{
Laszlo Göbölös, ${ }^{1,2}$ Simon Duggan, ${ }^{2}$ Laszlo \\ Lenard,' Reka Faludi,' Laszlo Hejjel' \\ 'Faculty of Medicine, University of Pecs, Hungary \\ ${ }^{2}$ Department of Cardiothoracic Surgery, Southampton \\ University Hospital Trust, United Kingdom
}

\author{
Correspondence: Laszlo Göbölös, Department of \\ Cardiothoracic Surgery, Southampton General Hospital, \\ Southampton University Hospital Trust, United Kingdom, Tel \\ +44-7756-384938, Fax +44-2380-794526, \\ Email isartor@hotmail.com
}

\section{Introduction}

Infective endocarditis is a fatal disease without adequate treatment. Right-sided endocarditis has a lower incidence than its left-sided counterpart. This is a reflection of the overall lower incidence of rightsided structural valvular deformities largely secondary to the reduced shear stress on the endocardial surface. ${ }^{1}$ Tricuspid valve (TV) infective endocarditis is a serious complication of intravenous drug abuse with a reported mortality between 5\% and 10\%. Staphylococcus aureusis the most frequent organism encountered and is associated with increased morbidity and higher mortality. ${ }^{2,3}$ In approximately half of the cases, $\mathrm{TV}$ endocarditis is the source of septic pulmonary emboli resulting in lung abscesses. Although potentially a life-threatening complication, in the absence of a severely affected TV, lung abscesses can be treated by medical management alone. ${ }^{4}$

\section{Case presentation}

We report a 30-year-old male, intravenous drug abuser, referred with tricuspid valve endocarditis and bilateral multiple lung abscesses (Figure 1A) (Figure 1B). Over the preceding 2-months, he developed insidious symptoms of fatigue and dyspnoea. A transthoracic echocardiogram demonstrated severe tricuspid regurgitation and blood cultures grew methicillin sensitive Staphylococcus aureus. Initial treatment included combined broad-spectrum antibiotics. The pulmonary abscesses resolved following a period of microbial containment and pre-operative stabilization. He underwent a tricuspid valve replacement (Figure 1C) with a low profile $31 \mathrm{~mm}$ mechanical prosthesis (ATS Open Pivot ${ }^{\circledR}$ Heart Valve, ATS Medical Inc, Minneapolis, USA) (Figure 1D). Following an uneventful postoperative course, the patient was discharged with endocarditis prophylaxis after 8 weeks and referred to a drugs rehabilitation facility. Three monthly follow-ups for 5years showed preserved bi-ventricular function with a functioning prosthesis at a constant mean gradient $<3 \mathrm{mmHg}$. During this period, the patient was free of narcotics and experienced no further pulmonary complications.

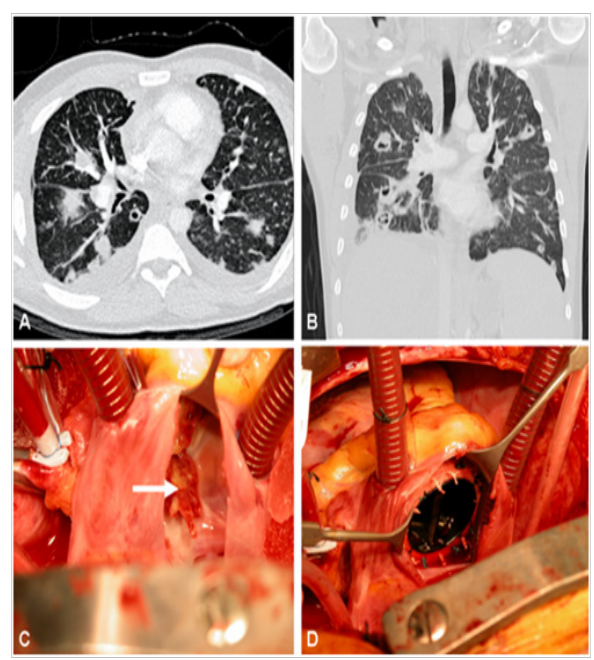

Figure IA CT chest showing multiple lung abscesses in the horizontal plane. Figure IB Multiple pulmonary abscesses in the coronal plane on CT chest. Figure IC Intraoperative view of the destroyed tricuspid valve (white arrow marks the floating mass).

Figure IDWell-seated low profile mechanical prosthesis in tricuspid position.

\section{Discussion}

Since the first description of malignant endocarditis in 1855 by Sir W Osler, the affected patient population and the causative organisms have significantly changed. ${ }^{5}$ Left sided endocarditis has a reported median incidence of 3.6/100,000 population per year, with a surge at the age of 65 years to $15.0 / 100,000$ per year and a male to female ratio of $2: 1$; right sided lesions occur in $5-10 \%$ of all endocarditis cases. ${ }^{6}$ In tricuspid valve endocarditis, the valve can be repaired in the majority of cases; only if the valvular structure is severely affected is a replacement required. Controversy exists as to the optimal timing of the surgery. Convention dictates that the disease initially be treated 
medically and to surgically reconstruct the valvular structures when possible in a non-infected environment. However, in the presence of resistant pathogens, multi-organ involvement or haemodynamic instability, expedited surgery is required despite the predicted higher postoperative morbidity and mortality. ${ }^{7}$ Surgical technique is also important.

Bauernschmitt et al. ${ }^{8}$ demonstrated that radical debridement of all the infected area is essential for successful long-term outcome. Furthermore, using this technique, the presence of a paravalvular abscess has no effect on outcome. ${ }^{9,10}$ Additionally, there appears to be no difference between valve repair and replacement. Musci et al. ${ }^{11}$ revealed that tricuspid valve reconstruction versus replacement has no statistically significant influence on early and late mortality. In their series??? The main causes of death were septic multi-organ failure, heart failure and haemorrhagic shock. ${ }^{11}$ Our brief report shows that TV infective endocarditis complicated by a severe secondary organ manifestation can be effectively treated. This requires multidisciplinary cooperation in the heart team to ensure early antimicrobials and correctly timed surgical intervention. Furthermore, a supportive family background, a proficient drugs rehabilitation programme and meticulous heart team follow-ups ensure a favorable outcome.

\section{Acknowledgements}

None.

\section{Conflict of interest}

The author declares no conflict of interest.

\section{References}

1. Nakagawa T, Wada H, Sakakura K, et al. Clinical features of infective endocarditis: Comparison between 1990 and 2000s. J Cardiol. 2014;63(2):145-148.

2. Sanfilippo AJ, Picard MH, Newell JB, et al. Echocardiographic assessment of patients with infectious endocarditis: prediction of risk for complications. J Am Coll Card. 1991;18(5):1191-1199.
3. Sande MA, Lee BL, Mills J, et al. Endocarditis in intravenous drug users. In: Kaye D editor. Infective endocarditis. 2nd ed. New York: Raven Press; 1992. 345 p

4. Baddour LM, Wilson WR, Bayer AS, et al. Infective endocarditis: diagnosis, antimicrobial therapy, and management of complications: a statement for healthcare professionals from the committee on rheumatic fever, Endocarditis, and Kawasaki disease, council on cardiovascular disease in the young, and councils on clinical cardiology, stroke, and cardiovascular surgery and anesthesia, American heart association: endorsed by the infectious diseases Society of America. Circulation. 2015;111(23):e394-e434.

5. Hoen B, Alla F, Selton-Suty C, et al. Changing profile of infective endocarditis: results of a 1-year survey in France. JAMA. 2002;288(1):75-81.

6. Mylonakis E, Calderwood SB. Infective endocarditis in adults. $N$ Engl $J$ Med. 2001;345(18):1318-1330.

7. Denk K, Vahl CF. Endocarditis: Entscheidungshilfen fur den optimalen Zeitpunkt zur operativen Sanierung. Herz. 2009;34(3):198-205.

8. Bauernschmitt R, Jakob HG, Vahl CF, et al. Operation for infective endocarditis: results after implantation of mechanical valves. Ann Thorac Surg. 1998;65(2):359-364.

9. Lange R, Simone R de, Bauernschmitt R, et al. Chirurgie der akuten Aortenklappenendokarditis: Prognose bei paravalvulären Abszessen. Z Kardiol. 1995;87(4):276-282.

10. Bauernschmitt R, Vahl CF, Lange R, et al. Surgical treatment of acute endocarditis of the aortic valve with paravavular abscess:considerations justifying the use of mechanical replacement devices. Eur J Cardiothorac Surg. 1996;10(9):741-747.

11. Musci M, Siniawski H, Pasic M, et al. Surgical treatment of rightsided active infective endocarditis with or without involvment of the left heart: 20-year single centre experience. Eur J Cardiothorac Surg. 2007;32(1):118-125. 